\title{
Preparation of a Thermo-sensitive Poly(lysine ester-co-adipic acid) with Excellent Biocompatibility
}

\author{
Zhihui Fan* ${ }^{1}$, Ting Jiang ${ }^{1}$, Chang Zheng ${ }^{1}$, Akoda Komlan Elom ${ }^{1}$, Jihua Song ${ }^{1}$ \\ ${ }^{1}$ College of Chemistry \& Environmental Science, Hebei University, Baoding 071002, China
}

\section{E-mail: 799509158@qq.com}

\begin{abstract}
Keywords: biocompatibility; thermo-sensitive polymer; lysine acid; molecular design Abstract: Good biocompatibility or no cytotoxicity is the most important characteristic for thermo-sensitive polymeric material because of its potential applications in the biomedical field. In this paper, a new poly (lysine ester-Co-adipic acid) (PLA) with both thermo-sensitivity and good biocompatibility was designed and successfully prepared. UV measurements indicated that PLA showed a reversible phase transition at $17.3-31.7^{\circ} \mathrm{C}$ (the lower critical solution temperature, LCST). LCST of PLA was affected by its molecular weight, structure and concentration. Moreover, the viability of HeLa cells after $24 \mathrm{~h}$ incubation in PLA solution was in the range of $98 \%-110 \%$, suggesting good biocompatibility of PLA. In summary, the new thermo-sensitive PLA with no cytotoxicity could be utilized as a promising biomaterial in the biomedical field.
\end{abstract}

\section{Introduction}

The interests in temperature-responsive polymers have persisted over several decades, and a great deal of work has been dedicated to developing thermo-sensitive macromolecules [1] which show a lower critical solution temperature (LCST) or the upper critical solution temperature (UCST) in aqueous solutions. Thermo-sensitive polymers are greatly useful in biomedical applications including drug delivery, tissue engineering, bio-separation and so on [2-3]. However, many common thermo-sensitive polymers are limited because of the lack of good biocompatibility. If the biocompatibility of the thermo-sensitive polymers is not improved, the thermo-sensitive polymers can only act as "smart materials" but not "safe materials". In order to improve the biocompatibility of thermo-sensitive polymers, researchers usually combine the known thermo-sensitive polymers with natural polymer or bio-micromolecule with biodegradability and biocompatibility [4] by pendant-group-modifying, blending, grafting, blocking, and cross-linking [5].

In this paper, our investigation was focused on the thermo-sensitivity and biocompatibility of the newly-synthesized polymeric materials. We designed and successfully prepared a new family of poly(lysine ester-CO-adipic acid) (PLA) with excellent biocompatibility and thermo-sensitivity. Results indicated that PLA showed a reversible phase transition at $17.3-31.7^{\circ} \mathrm{C}$. In addition, the high viability of HeLa cells was found to exceed $90 \%$ after incubation for $24 \mathrm{~h}$. Briefly, PLA with outstanding thermo-sensitivity and biocompatibility was likely to become a promising material in the biomedical fields.

\section{Experiment}

Materials and Methods. $L$-lysine hydrochloride, methanol ethanol, and 1-propanol were received from Kermel Reagent Factory (Tianjin, China) with analytical grade. Sodium chloride and triethylamine were purchased from Guangfu Fine Chemical Factory (Tianjin, China). Ether and triethylamine (TEA) were purified by drying and distillation before using. The other chemicals were also analytical grade and used without any further purification. Moreover, HeLa cells were purchased from Peking Union Medical College Hospital.

Synthesis of poly(lysine methyl ester-co-adipic acid) (PLMA). In a representative procedure, $L$-lysine methyl ester hydrochloride $(0.6993 \mathrm{~g}, 3 \mathrm{mmol})$ was prepared according to the reported method [6] and triethylamine (12 mmol) were dissolved in methanol, and this solution was stirred in the ice water bath for $20 \mathrm{~min}$. After the mixture dissolved completely, adipoyl chloride $(0.5490 \mathrm{~g}$, $3 \mathrm{mmol}$ ) was added into the tube. The reaction was kept on $3 \mathrm{~h}$ with vigorous stirring at $0^{\circ} \mathrm{C}$. After 
that, the solution was dropwise added into the saturated salt water $(30 \mathrm{~mL})$, to precipitate out of the crude PLMA. $30 \mathrm{~mL}$ methanol was added into the tube to dissolve the product, and the polymer solution was dropped slowly into ether to precipitate the resulting polymer. By repeating the dissolution and precipitation processes, the target polymer was got and then dried to a constant weight under vacuum at $50^{\circ} \mathrm{C}$. Similarly, poly(lysine ethyl ester-Co-adipic acid) (PLEA) and poly(lysine propyl ester-CO-adipic acid) (PLPA) could be synthesized with the aforementioned process. Fig. 1 was the synthesis route of thermo-sensitive PLA from $L$-lysine ester hydrochloride and adipoyl chloride.

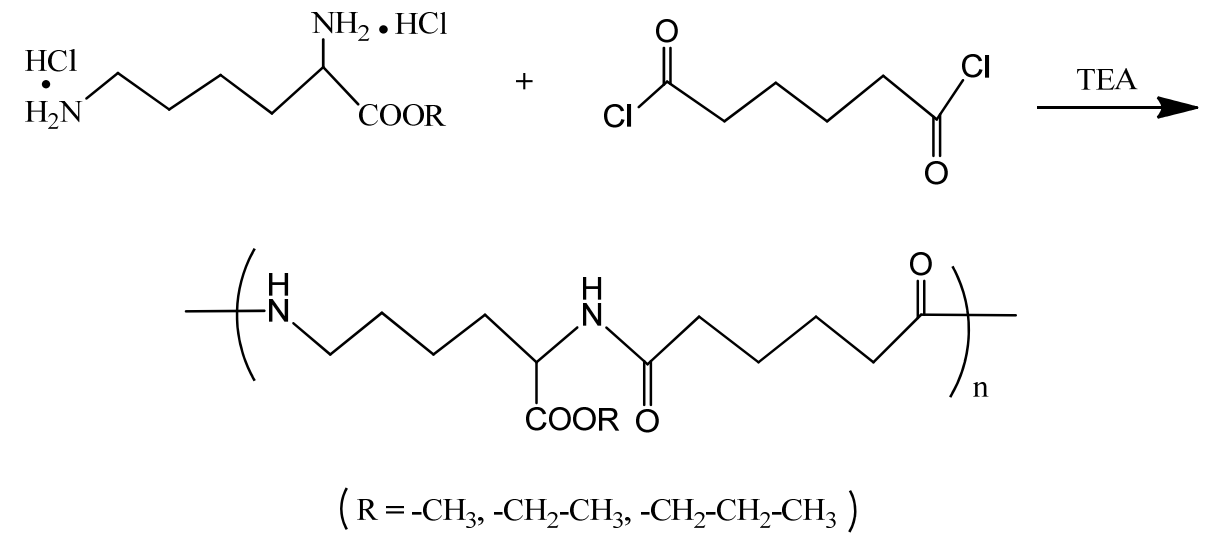

Fig. 1 Schematic synthesis of thermo-sensitive PLA with biocompatibility

\section{Results and Discussion}

Structural Characterization of PLMA. As a representative for PLA, the chemical structure of PLMA was certificated by FTIR, ${ }^{1}$ H NMR spectrum and GPC measurement. The FTIR spectrum of PLMA was showed in Fig. 2. The amide linkage $(\mathrm{N}-\mathrm{H})$ stretching vibration and deforming vibration in the amide were found at $3297 \mathrm{~cm}^{-1}$ and $1545 \mathrm{~cm}^{-1}$, respectively. The signal at $1740 \mathrm{~cm}^{-1}$ was contributed to the stretching vibration of carbonyl $(\mathrm{C}=\mathrm{O})$ in the ester. A band at $1648 \mathrm{~cm}^{-1}$ was ascribed to the stretching vibration of carbonyl $(C=O)$ in amide group. The peak at $2938 \mathrm{~cm}^{-1}$ was the stretching vibration of the group $\mathrm{C}-\mathrm{H}$ in the polymer. Furthermore, the ether linkage $(\mathrm{C}-\mathrm{O}-\mathrm{C})$ in the ester can be discovered at $1190 \mathrm{~cm}^{-1}$.

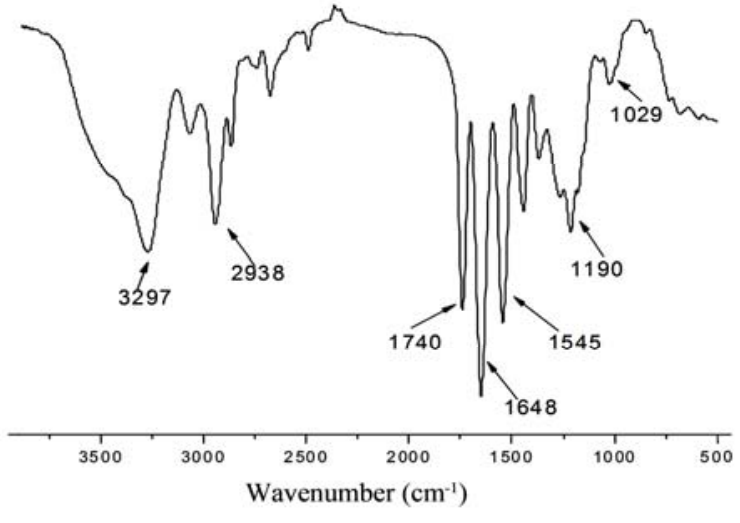

Fig. 2 FTIR spectrum of PLMA

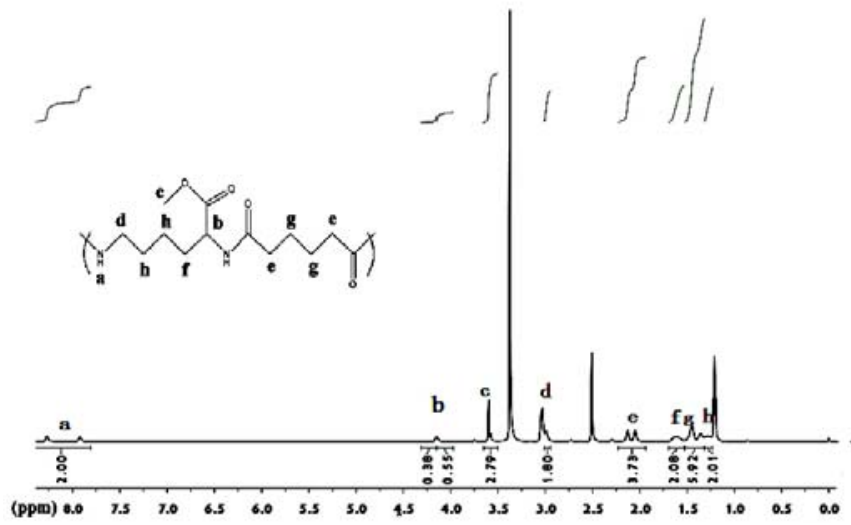

Fig. $3{ }^{1} \mathrm{H}$ NMR spectrum of PLMA (In DMSO $-d 6$ )

${ }^{1} \mathrm{H}$ NMR spectrum of the PLMA in deuterated DMSO was shown in Fig. 3. The obvious peak ascribing to N-H from the PLMA was observed at $8.0 \mathrm{ppm}$. The characteristic peak of $\mathrm{H}$ atom from the tertiary carbon in the main chain was recorded at $4.14 \mathrm{ppm}$ in Fig. 3. The signal at $3.60 \mathrm{ppm}$ was ascribed to the lysine methyl ester structural unit. The symbol at $3.03 \mathrm{ppm}(\mathrm{d})$ was attributed to $\mathrm{H}$ on the secondary carbon of lysine methyl ester structural unit next to the N-H. The other $\mathrm{H}$ atoms from methyl and methylene could be found in the spectrum, which ranged from $1.00 \mathrm{ppm}$ to 2.50 ppm. In addition, the ratio among hydrogen atoms was in good agreement with the chemical 
structure of PLMA. All these data were enough to prove that the PLMA has been synthesized successfully in our experiment. Similarly, the chemical structure of PLEA and PLPA was confirmed by the FTIR and ${ }^{1} \mathrm{H}$ NMR measurements.

The Mn of PLMA reaches its highest value $\left(\mathrm{Mn}=2.95 \times 10^{4} \mathrm{~g} / \mathrm{mol}\right.$ in the GPC measurement), when the ratio of two monomers equals 1:1. However, the polymer molecular weights of other ratios deceased to $2.92 \times 10^{4} \mathrm{~g} / \mathrm{mol}$ and $1.24 \times 10^{4} \mathrm{~g} / \mathrm{mol}$, as the ratio of adipoyl chloride was changed to 0.95 and 1.05 respectively. In addition, there was little difference between the polydispersity index, which all range from 1.82 to 2.12 of the three polymers.

Effect of structure of PLA on LCST. In the study, the transmittance of pure water in the corresponding temperature was defined as $100 \%$. Fig. 4 depicted the phase transition processes of PLMA and PLEA at wt $1.2 \%$. The transmittance of PLEA solution decreased from $94.2 \%$ at $-11^{\circ} \mathrm{C}$ to a constant level of $3.8 \%$ at $17{ }^{\circ} \mathrm{C}$, the transmittance of PLMA solution undergone an abrupt change from $90.3 \%$ at $12{ }^{\circ} \mathrm{C}$ to $20.6 \%$ at $42{ }^{\circ} \mathrm{C}$ in the heating run. Obviously, the LCST of PLEA was much lower than PLMA. When tried to increase one more hydrophobic group as PLPA, the thermo-responsive behavior could not be achieved. It was similar to the behaviors of some thermo-sensitive polymers such as poly (vinylphosphonate) [7], hyperbranched polyethers [8] et al. This phenomenon was attributed to the one more hydrophobic group $\left(-\mathrm{CH}_{2}-\right)$ in the PLEA than PLMA, which endowed more hydrophobic nature to PLEA. Therefore, PLEA needed lower temperature to remove the interactions between macromolecular chains and water molecules.

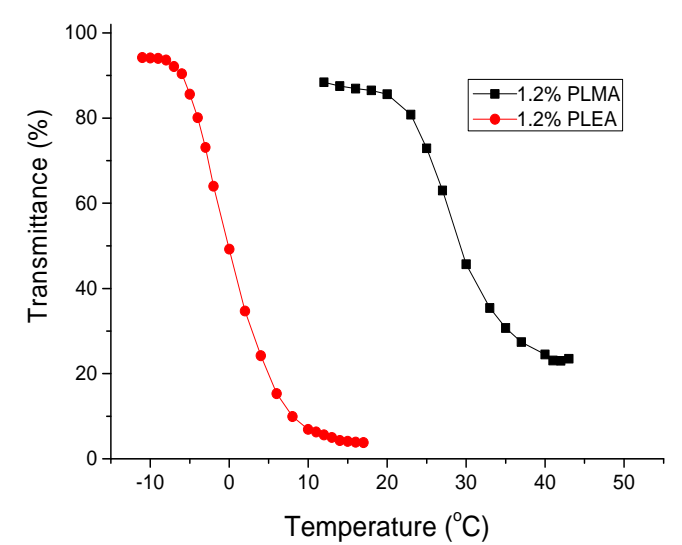

Fig. 4 The phase transition curves of PLMA and PLEA

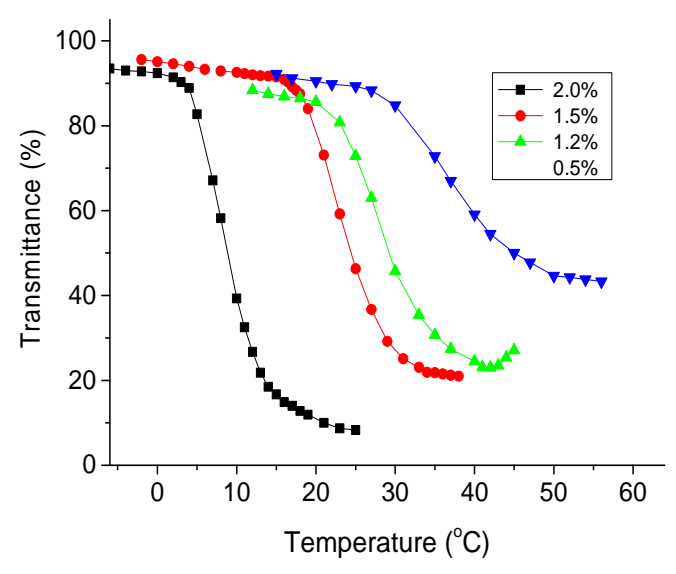

Fig. 5 The phase transition curves of PLMA with different concentrations

Effect of Concentration of PLMA on LCST. Fig. 5 displayed the relation of transmittance and temperature of PLMA solution with different concentration. The phase transition of $2.0 \mathrm{wt} \%$ started at $4{ }^{\circ} \mathrm{C}$ with nearly $90 \%$ transmittance and ended at $21^{\circ} \mathrm{C}$ with $10.4 \%$ transmittance, which the phase transition range was about 17 degree. However, when the concentration decreased to $0.5 \mathrm{wt} \%$, the phase transition expanded to 23 degree. The transition zone became broader with the concentration decreasing, which was consistent with the results reported by Wang [10]. According to LCST calculating method by Wang [9], the LCST of PLMA was calculated about $40{ }^{\circ} \mathrm{C}$ when polymer concentration was extrapolated to zero.

Cytotoxicity evaluation for PLMA, PLEA and PLPA. Cytotoxicity of PLA to HeLa cells was assessed by MTT method. As shown in Fig. 6, when the concentration of PLMA, PLEA, and PLPA varied from 1 to $1000 \mu \mathrm{g} \cdot \mathrm{mL}^{-1}$, the cell viability was higher than $105 \%$ after $24 \mathrm{~h}$ incubation. Especially, the cell viability increased more than $110 \%$ when the concentrations of PLMA and PLEA were 10, 100 and $1000 \mu \mathrm{g} \cdot \mathrm{mL}^{-1}$. The cell viability at the concentration value "Control" was used as reference which meant the cells growth in natural situation. Obviously, the cell viability in presence of PLA was much better than the viability in natural conditions. Accordingly, PLMA, PLEA and PLPA materials showed no cytotoxicity and were highly biocompatible for living cells. 
The excellent biocompatibility of PLMA, PLEA and PLPA exactly resulted from lysine units in the molecular chains.

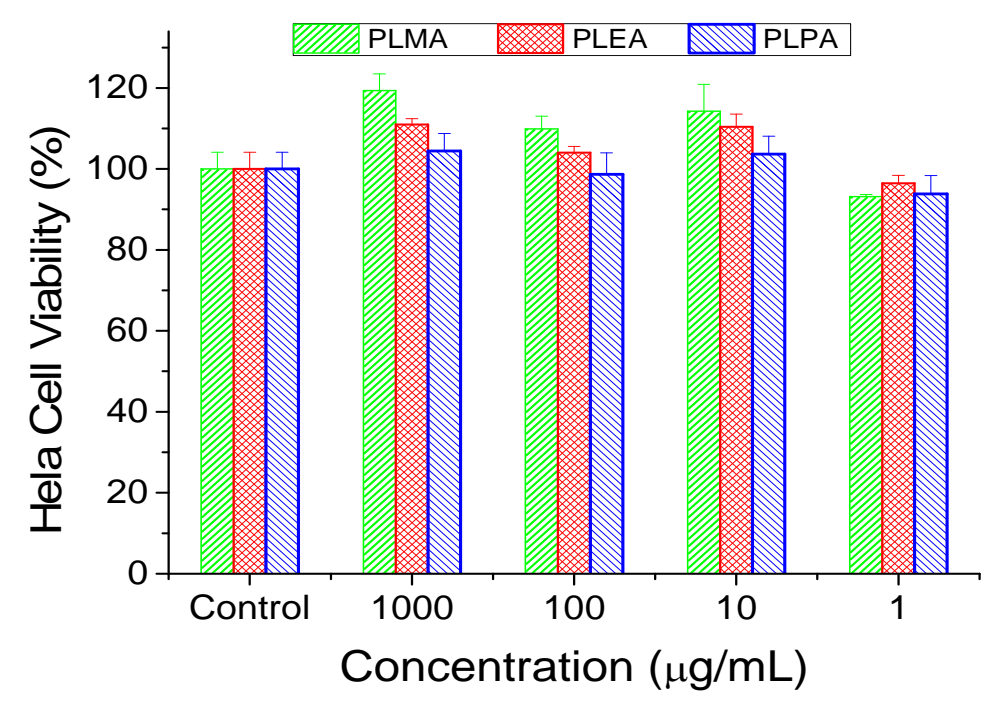

Fig. 6 Viability of HeLa cells of PLA after $24 \mathrm{~h}$ incubation.

\section{Conclusions}

In this study, we designed and successfully prepared a novel poly(lysine ester-co-adipic acid) (PLA) with good thermo-sensitivity and biocompatibility. Thermo-sensitivity of PLA was regulated via the adjustment of alkyl group in ester moiety, and their reversible LCST phase transition was found to be at $17.3-31.7{ }^{\circ} \mathrm{C}$. More importantly, the viability of HeLa cells was higher than $90 \%$ after $24 \mathrm{~h}$ incubation in PLA solution, implying excellent biocompatibility. The thermo-sensitive PLA with excellent biocompatibility can be used as promising materials in the biomedical field.

\section{Acknowledgements}

The research was financially supported by Training Program for Innovative Research Team and Leading talent in Hebei Province University (Grant No: LJRC024); Key project of Hebei Education Department (Grant No: ZD20131047).

\section{References}

[1] M. Burek, Z.P. Czuba and S. Waskiewicz: Polymer. Vol.55 (2014), p. 6460

[2] A. Galperin, T.J. Long and B.D. Ratner: Biomacromolecules. Vol.11 (2010), p. 2583

[3] S. Champ, W. Xue and M.B. Huglin: Polymer.Vol.42 (2011), p. 6439

[4] D.L. Huber, R.P. Manginell, M.A. Samara, B.I. Kim and B.C. Bunker: Science.Vol.301 (2003), p. 352

[5] J. Reguera, J.M. Lagaron, M. Alonso, V. Reboto, B. Calvo and J.C. Rodríguez-Cabello: Macromolecules Vol.36 (2003), p. 8470

[6] H.Y. Deng, Z.M. Yin, T. Jiang, H.M. Liu, X.Y. Fan, M. Wang, X. Ma, Z.H. Fan, C. Zheng and K.L. Deng: Colloid Polym Sci.Vol.293 (2015), p. 2341

[7] N. Zhang, S. Salzinger and B. Rieger: Macromolecules. Vol.45 (2012), p. 9751

[8] Z.F. Jia, H. Chen, X.Y. Zhu and D.Y. Yan: J. Am. Chem. Soc. Vol.128 (2006), p. 8144

[9] X.H. Wang, X.P. Qiu and C. Wu: Macromolecules Vol.31 (1998), p. 2972 